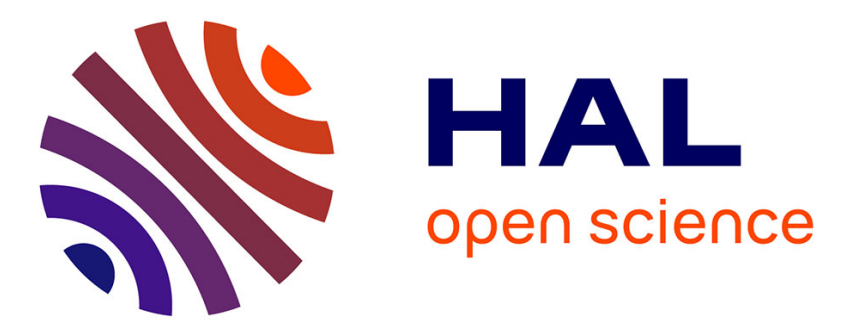

\title{
Diversification des modèles de qualité territorialisée des produits agroalimentaires : risque ou opportunité pour les terroirs?
}

Michaël Pouzenc, Dominique Coquart, Jean Pilleboue, Valérie Olivier, Martine Guibert

\section{To cite this version:}

Michaël Pouzenc, Dominique Coquart, Jean Pilleboue, Valérie Olivier, Martine Guibert. Diversification des modèles de qualité territorialisée des produits agroalimentaires: risque ou opportunité pour les terroirs?. Méditerranée: revue géographique des pays méditerranéens, 2007, 109, pp.31-40. halshs-00697840

\section{HAL Id: halshs-00697840 \\ https://shs.hal.science/halshs-00697840}

Submitted on 16 May 2012

HAL is a multi-disciplinary open access archive for the deposit and dissemination of scientific research documents, whether they are published or not. The documents may come from teaching and research institutions in France or abroad, or from public or private research centers.
L'archive ouverte pluridisciplinaire HAL, est destinée au dépôt et à la diffusion de documents scientifiques de niveau recherche, publiés ou non, émanant des établissements d'enseignement et de recherche français ou étrangers, des laboratoires publics ou privés. 


\title{
Méditerranée
}

Numéro 109 (2007)

Terroirs : caractérisation, développement territorial et gouvernance

Michaël Pouzenc, Dominique Coquart, Jean Pilleboue, Valérie Olivier et Martine Guibert

\section{Diversification des modèles de qualité territorialisée des produits agroalimentaires : risque ou opportunité pour les terroirs?}

\begin{abstract}
Avertissement
Le contenu de ce site relève de la législation française sur la propriété intellectuelle et est la propriété exclusive de l'éditeur.

Les œuvres figurant sur ce site peuvent être consultées et reproduites sur un support papier ou numérique sous réserve qu'elles soient strictement réservées à un usage soit personnel, soit scientifique ou pédagogique excluant toute exploitation commerciale. La reproduction devra obligatoirement mentionner l'éditeur, le nom de la revue, l'auteur et la référence du document.

Toute autre reproduction est interdite sauf accord préalable de l'éditeur, en dehors des cas prévus par la législation en vigueur en France.
\end{abstract}

\section{revues.org}

Revues.org est un portail de revues en sciences humaines et sociales développé par le Cléo, Centre pour l'édition électronique ouverte (CNRS, EHESS, UP, UAPV).

\section{Référence électronique}

Michaël Pouzenc, Dominique Coquart, Jean Pilleboue, Valérie Olivier et Martine Guibert, « Diversification des modèles de qualité territorialisée des produits agroalimentaires : risque ou opportunité pour les terroirs ? », Méditerranée [En ligne], 109 | 2007, mis en ligne le 01 juillet 2009. URL : http://mediterranee.revues.org/111 DOI : en cours d'attribution

Éditeur : Publications de l'université de Provence http://mediterranee.revues.org http://www.revues.org

Document accessible en ligne sur : http://mediterranee.revues.org/111 Ce document est le fac-similé de l'édition papier.

Tous droits réservés 


\title{
Diversification des modèles de qualité territorialisée des produits agroalimentaires: risque ou opportunité pour les terroirs?
}

\author{
Diversification of models of territorialized quality of foods products: risk or opportunities \\ for the terroirs?
}

\author{
Michaël POUZENC \\ enseignant-chercheur \\ UMR « Dynamiques Rurales » \\ UTM, ENFA, INPT-ENSAT \\ Toulouse, France \\ pouzenc@univ-tlse2.f
}

\author{
Dominique COQUART \\ enseignant-chercheur \\ UMR « Dynamiques Rurales » \\ UTM, ENFA, INPT-ENSAT \\ Toulouse, France \\ coquart@ensat.fr \\ Valérie OLIVIER \\ enseignante-chercheur \\ UMR « Dynamiques Rurales » \\ UTM, ENFA, INPT-ENSAT \\ Toulouse, France \\ olivier@ensat.fr
}

\author{
Jean PILLEBOUE \\ enseignant-chercheur \\ UMR « Dynamiques Rurales » \\ UTM, ENFA, INPT-ENSAT \\ Toulouse, France \\ pillebou@univ-tlse2.fr
}

\section{Martine GUIBERT \\ enseignante-chercheur UMR « Dynamiques Rurales » UTM, ENFA, INPT-ENSAT Toulouse, France guibert@univ-tlse2.fr}

\begin{abstract}
Résumé - À côté du «modèle AOC», parfois en liaison avec lui, se multiplient d'autres démarches de territorialisation de la qualité, utilisant des références variées aux éléments constitutifs du terroir et, plus largement, du territoire. À partir de diverses études de cas, nous proposons d'organiser cette diversité d'initiatives autour de trois « pôles » d'attentes des producteurs et des consommateurs et autour de cinq principaux «modèles» de qualité territorialisée. Nous précisons ensuite pour quelles raisons le « modèle AOC 》 peut rencontrer différents risques et opportunités : qu'elles soient convergentes ou divergentes, les démarches de qualité territorialisée évoluent sans cesse et la place dévolue au terroir y est constamment remise en question.
\end{abstract}

\section{I - Introduction}

Jusqu'en 1990, la reconnaissance de l'AOC, par le lien qu'elle établit entre une aire de production et un produit agricole, suffisait à distinguer celui-ci de tous les autres produits du même type. Depuis lors, la perception de cette référence a été obscurcie par la multiplication des démarches qui affichent un lien au territoire par d'autres voies. Ce mouvement s'analyse d'abord comme une étonnante accélération des processus. Alors qu'il avait fallu presque un siècle pour que l'AOC soit bien définie et reconnue socialement, une ou deux décennies pour que soit complété le système des signes officiels de qualité et d'origine (label rouge, IGP), quelques années suffisent maintenant pour que de nouvelles voies de différenciation, plus ou moins clairement référées à un territoire, parviennent à la notoriété commerciale. Voilà donc qui ne peut être correctement apprécié sans être replacé dans la perspective d'un temps long, tout comme dans celle de l'élargissement de l'horizon géographique dans lequel la qualification des produits s'effectue et prend tout son sens. Ensuite, alors que les producteurs agricoles ont longtemps été les principaux acteurs de la qualification territoriale de leurs produits, ils ont été rejoints voire supplantés dans cette fonction par les autres acteurs des filières agroalimentaires (industriels, distributeurs et publicitaires), par les collectivités territoriales et, de plus en plus, par « les consommateurs » eux-mêmes. Enfin, quels qu'en
Abstract - Nearby the "geographic indications model», sometimes linked to it, other territorial quality initiatives are manifolding, using various references to "terroir" and, more generally, to territory. Based on diversified case studies, we propose to organise this initiatives diversity around three producers and consumers " poles » of expectations and around five territorial quality "models". Then we precise the reasons why the "geographic indications model » may meet some risks and opportunities: converging or diverging, territorial quality initiatives are continuously changing and each time the place dedicated to "terroir" is reassessed.

soient les auteurs, le corpus qualifiant des produits s'est lui-même élargi et complexifié. Tandis que les signes officiels de qualité et d'origine organisent une relation au territoire par un long apprentissage entre le produit et «son» terroir, d'autres variables (proximité versus éloignement ou exotisme, valeurs éthiques ou citoyennes, ...) ont fait irruption dans les processus les plus récent de qualification. Au terroir, envisagé comme creuset d'un milieu physique et d'une histoire sociale et culturelle, s'ajoutent d'autres dimensions du territoire, envisagé comme produit de constructions sociales qui mettent l'espace en jeu.

Examinons donc les nouvelles formes que prend le territoire dans ces configurations, ainsi que la place accordée au terroir. Quels sont ses «nouveaux habits», quels liens nouveaux se tissent entre terroirs, produits et consommateurs et à quelles échelles ? Comment ordonner et éclairer ces complexités qui résultent de la prolifération des références au territoire et du fait que les nouvelles s'ajoutent aux anciennes sans en rendre aucune caduque ?

Comme nous y a invité l'appel à communication, notre réflexion est pluridisciplinaire: elle a associé géographes et économistes dans l'étude de cas aussi divers que possible. Nous avons comparé les démarches des différents acteurs de la promotion des produits agroalimentaires dits «de qualité », en particulier du point de vue «des 
attentes " qu'ils prêtent aux consommateurs. À l'issue de cette phase de travail empirique, il nous semble que la diversité des initiatives de qualité territorialisée peut s'organiser autour de trois types d'attentes des producteurs et des consommateurs (partie I) et que les réponses apportées à ces attentes relèvent principalement de cinq «modèles", déclinés de multiples manières (partie II). Les initiatives de qualité territorialisée semblent en outrese renouveler continuellement, en empruntant aux différents modèles; qu'elles soient convergentes ou divergentes, la place dévolue au terroir y est sans cesse remise sur le métier. Le recours à la théorie économique nous permet d'interpréter ce renouvellement (partie III). Risque ou opportunité pour les terroirs? Telle est bien la question qui guide notre réflexion.

\section{2 - Les trois pôles de la qualité agroalimentaire}

Les attentes vis-à-vis de la qualité des produits s'inscrivent nous semble-t-il dans trois registres. Dans le premier, producteurs et consommateurs s'attachent surtout aux liens qui unissent les caractéristiques des produits à celles des territoires de production. Le produit est alors à la fois « inimitable » et « emblématique » d'un territoire. Dans le deuxième registre, l'attention porte davantage sur le respect de normes techniques propres à garantir la composition physico-chimique des produits, leur fiabilité et la sécurité sanitaire. Le dernier registre correspond aux attentes de plus en plus souvent rassemblées sous le vocable «développement durable», combinant diverses préoccupations d'ordre éthique. En fonction de ces grands types d'attentes, nous pouvons dessiner trois «pôles» de la qualité agroalimentaire territorialisée, les pôles «terroir », « mode de production » et « citoyen » (fig. 1).

\section{LES MODÈLES DE LA QUALITÉ AGROALIMENTAIRE}

\section{PÔlE \\ "MODE DE PRODUCTION"}

Attentes $=$ respect des normes techniques,

fiabilité, sécurité sanitaire

Territoire $=$ milieu physique et $/ \mathrm{ou}$

bassin de production et/ou

système productif localisé

Attentes = respect de procédés traditionnels, authenticité, origine

Territoire $=$ terroir, relation à un patrimoine perpétué

\section{PÔLE} "TERROIR"

LIEN AU TERRITOIRE :
Attentes $=$ respect des principes

du développement durable,

solidarité, proximité, éthique

Territoire $=$ environnement et $/ \mathrm{ou}$ rapports sociaux localisés

PÔLE "CITOYEN" 


\section{I - Le pôle « terroir »}

Ici, les consommateurs attendent de retrouver, à travers les produits, une typicité considérée comme indissolublement liée à tout ce qu'ils se représentent comme étant les caractéristiques globales d'un espace géographique délimité, d'un terroir. Toutefois, contrairement à toutes les autres, les démarches de qualité territorialisée focalisées sur le terroir se développent dans une grande indépendance vis-à-vis des «attentes des consommateurs ». Portées par des «fabricants» qui sont le plus souvent des agriculteurs mais peuvent être, dans le cas de certaines AOC et de nombreuses IGP, des industriels ou des artisans, elles visent d'abord la protection d'une filière face à ses concurrentes.

Deuxième différence importante par rapport à toutes les autres initiatives, qui n'ont pas encore d'histoire, les démarches «terroir» dont les AOC françaises constituent l'archétype, sont «tannées par le temps» et il faut envisager leur genèse progressive tout au long d'un siècle, en particulier pour ce qui est des relations entre terroir et produit. Il va de soi qu'en même temps qu'on considère la façon dont ce type de démarche a gagné successivement diverses productions agricoles, il faudrait aussi prendre en compte la dimension territoriale de ces productions et bien noter dans quelles régions se sont développées les AOC. Rappelons également par quelles voies est passée la notion de «terroir» pour arriver, finalement, à rencontrer celles de «qualité » des produits, d'origine. Pendant longtemps, le mot a été attaché de façon péjorative à tout ce qui provenait des campagnes «profondes». Le contenu péjoratif de la notion s'est effacé - il a fallu plus d'un siècle jusqu'à s'inverser et à devenir un qualificatif valorisant.

Quels sont les invariants inséparables de la constitution des premières appellations d'origine et qui se sont maintenus tout au long de leur siècle d'histoire?

- Un collectif ...

- ...organisé pour la défense d'un produit de ses adhérents (défense de sa notoriété, d'une position commerciale acquise, de pratiques productives, etc.)

- Une argumentation par laquelle s'établit le lien $\mathrm{du}$ produit avec la notion de terroir, dont il se veut l'émanation exclusive. La référence au «terroir» se veut l'arme qui tue ... le concurrent dans la mesure où elle postule que tout terroir est unique. Cette exclusivité peut être, au choix du collectif, définie par des arguments de caractère naturaliste (sol, climat local, abri, orientation, position géographique, etc...) ou par la mise en exergue de pratiques socio-techniques avérées et validées par une certaine durée d'effectivité (les fameux « usages locaux, loyaux et constants»). Quoi qu'il en soit, elle se concrétise dans un cahier des charges.

- Une aire délimitée, une délimitation qui sanctionne l'exclusion de ce qui est «dehors ». L'aire de production ainsi définie se veut homogène mais elle ne l'est qu'envisagée $\mathrm{du}$ point de vue d'un seul critère (le sol, ou le climat, ou telle pratique, ou ...); pour le reste, elle est évidemment hétérogène.
- Un nom ... de lieu, de région, de village, de ville, etc. Bref « un nom géographique »!

- Une reconnaissance et une garantie institutionnelles : depuis 1935, elles sont assurées par l'INAO, organisme public sous tutelle de l'État.

- Le contrôle du respect des engagements productifs inscrits dans le cahier des charges.

Tous ces points font débat au moment où sont définies les appellations d'origine, qui émergent souvent après de terribles conflits socio-géographiques. Ceux-ci cristallisent sur le nom, sur la reconnaissance des caractères physiques particuliers du terroir et davantage encore sur les pratiques et savoir-faire ainsi que sur le tracé des limites. L'ouverture de l'AOC à d'autres produits que les produits viniques (vins et eau-de-vie) et les produits laitiers ne date que de 1990. Elle est allée de pair avec la mise en avant de la défense des petits producteurs et « la promotion du développement des régions en difficulté ». Jusque-là, les AOC étaient souvent promues, d'abord par des notables agricoles et ruraux, puis par des producteurs qu'on peut qualifier de « modernistes ». Il faut souligner que les thèmes «environnementaux» et «éthiques » n'ont pas encore acquis clairement droit de cité dans les procédures de définition des AOC.

Comme l'évoque le gradient de la figure 1, le lien au territoire est toujours important dans ce premier pôle. Le territoire y est évidemment considéré en tant que « terroir » ou assemblage de terroirs et comme médiateur d'une relation à une globalité (physique, historique, économique, sociale ...). L'échelle territoriale privilégiée est généralement locale, voire régionale, depuis les AOC limitées à un coteau viticole bien exposé (cas de l'AOC "Côte rotie »), jusqu'à celles qui recouvrent plusieurs régions agricoles (cas de l'Armagnac).

C'est à l'aune de chacun de ces éléments structurants que peuvent s'analyser les nouvelles formes de lien au terroir, ou de façon plus générale, de lien au territoire.

\section{2 - Le pôle « mode de production »}

Les produits qui se revendiquent « issus de l'agriculture biologique» ou « issus de l'agriculture raisonnée », « traditionnels », « fermiers », « artisanaux », sont extrêmement nombreux, tout autant que ceux qui affichent des messages rassurant le consommateur quant à leurs qualités organoleptiques ou sanitaires («sans traitement après récolte », «élevé en plein air », « riche en omégas $3 »$, etc.). Ces mentions renvoient toutes à l'idée que la qualité du produit est liée à la manière de produire. Le lien entre la qualité et le mode de production peut être explicité et garanti par un tiers, par exemple dans le cas des produits biologiques, pour lesquels il s'agit d'interdire certaines pratiques culturales (usage d'intrants chimiques). Le traditionnel, le fermier, l'artisanal restent pour leur part des notions floues, qui évoquent les savoir-faire transmis, la transformation dans l'exploitation, la petite quantité et le non standardisé. À l'opposé, pour les produits industriels, fabriqués en masse et de manière standardisée, sont développés des argumentaires basés sur l'innovation 
et le respect de normes techniques, garantissant la maîtrise des process et la composition du produit.

Ce pôle de qualification par le mode de production est aussi ancien. Ses liens au territoire sont variables, parfois aussi forts que dans le pôle «terroir», parfois beaucoup plus ténus. Dans ce cas, le territoire peut être perçu en tant que «milieu physique », c'est-à-dire comme un complexe topographique, pédologique, hydrologique et climatique, responsable pour partie de la composition physico-chimique des produits agricoles qui en sont issus. Ceci rejoint partiellement la notion de terroir. Le territoire peut également y être considéré en tant que «mode d'organisation d'un système d'acteurs », du type SPL (système productif local), c'est-à-dire comme un système socioéconomique local et relativement spécialisé, au sein duquel les savoirs, savoir-faire et coordinations participent à la construction d'un produit aux qualités spécifiques. L'échelle territoriale privilégiée est celle des bassins de production.

\section{3 - Le pôle « citoyen »}

Les produits « éthiques », les produits issus du commerce équitable ou solidaire, ou encore la multiplication des AMAP (Associations pour le Maintien de l'Agriculture Paysanne), témoignent de l'apparition de nouvelles demandes: certains consommateurs réclament des produits dotés d'attributs citoyens ou éthiques. Respecter les droits de l'homme, les droits sociaux, les droits environnementaux, voire le bien-être animal, acheter à un prix «juste», contribuer au maintien d'une petite agriculture paysanne, favoriser la «relocalisation» de l'économie participe à la construction d'une nouvelle qualité des produits agroalimentaires, basée sur la défense d'un certain nombre de valeurs.

Le développement de ces préoccupations citoyennes est assez tardif. Certes, les premières initiatives qui les prennent en compte datent déjà de plusieurs décennies. Elles ont progressé parallèlement à la conceptualisation du développement durable, depuis ses prémices des

\section{LES MODÈLES DE LA QUALITÉ TERRITORIALISÉE}

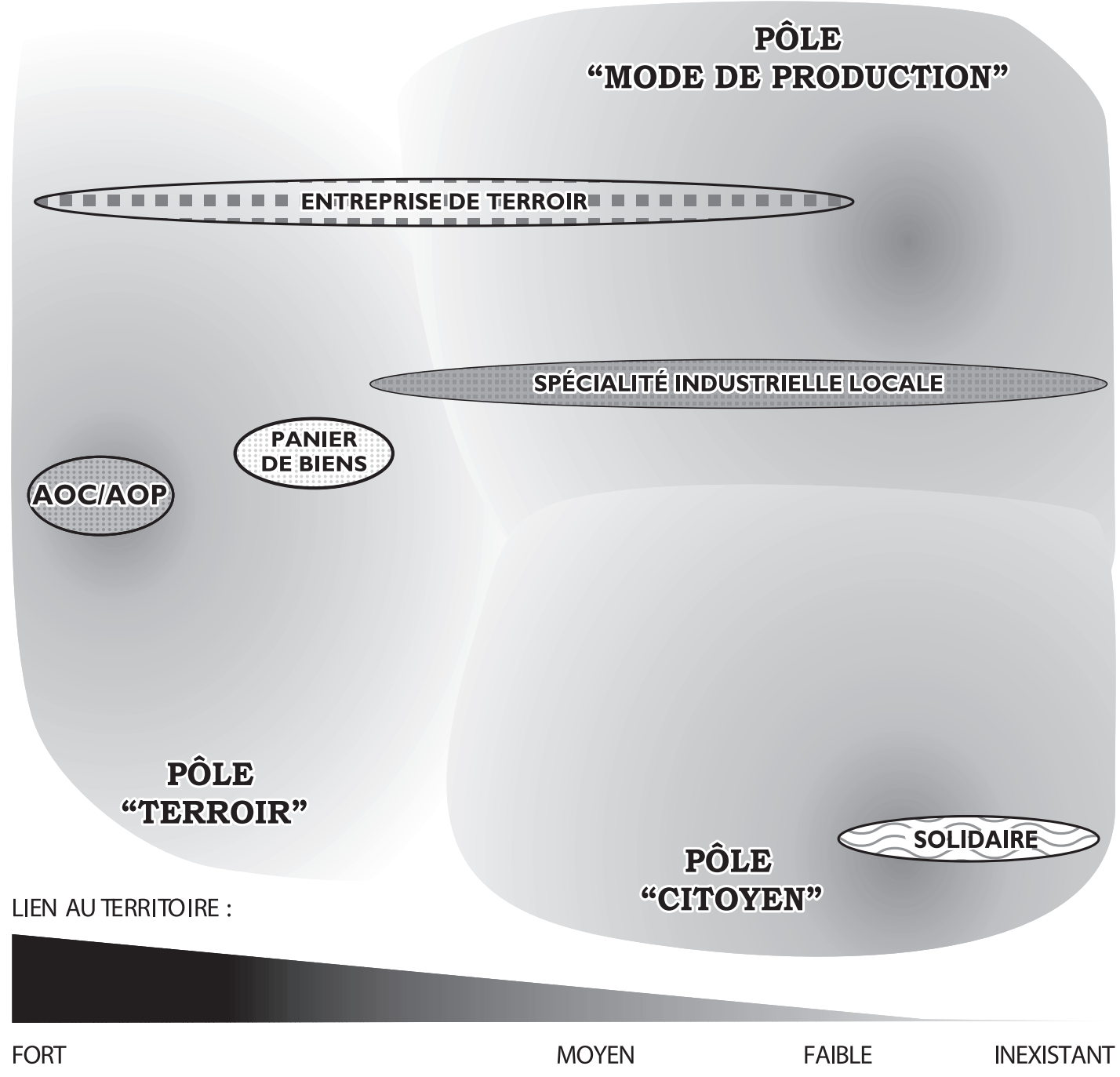


années 1950 jusqu’à sa formalisation des années 1980. Toutefois, la structuration des démarches « citoyennes » et surtout leur succès auprès des consommateurs sont beaucoup plus récents. Si l'association Max Havelaar, qui porte aujourd'hui le principal label de commerce équitable, est créée dès 1988, il faut attendre la fin des années 1990 pour que les produits du commerce équitable représentent une part de marché non négligeable. Autre exemple, le réseau régional des AMAP, intitulé « Alliance Midi-Pyrénées », n’existe que depuis 2004.

Dans ce pôle de préoccupations, les liens au territoire sont variables, jusqu'à être parfois inexistants. Ils sont de nature particulière. D'une part, le territoire peut y être considéré en tant qu' « environnement», c'est-à-dire comme un ensemble de caractéristiques écologiques à préserver. D'autre part, il peut être défini en tant que "proximité », c'est-à-dire comme un réseau de rapports sociaux localisés, rassemblant des producteurs et des consommateurs qui partagent le même ensemble de valeurs, qu'il s'agisse de personnes appartenant à une même localité (AMAP) ou de personnes géographiquement très éloignées (producteurs des pays du Sud et consommateurs des pays du Nord). Enfin, l'éventail d'échelles territoriales prises en compte est extrêmement large, depuis l'échelle locale lorsqu'il s'agit de préserver les zones agricoles entourant son agglomération jusqu'à l'échelle planétaire lorsqu'il s'agit de préserver la biodiversité ou d'améliorer les conditions de travail dans les pays du Sud.

Autour des trois grands types d'attentes ainsi présentés se développe une grande diversité d'initiatives.

\section{3 - Différents modèles de qualité territorialisée}

Nous proposons de classer ces initiatives foisonnantes en construisant une typologie de cinq «modèles» de la qualité territorialisée et d'interroger le rapport que chacun d'eux entretient avec le terroir (fig. 2).

Rappelons tout d'abord que le modèle «AOC », par son ancienneté, sa codification et les tentatives d'imitation qu'il suscite, est emblématique de ces démarches. Il prend en compte les différentes dimensions de l'insertion du produit dans le territoire: lien entre les conditions de milieu et les caractéristiques intrinsèques du produit, ancienneté de l'inscription territoriale, mobilisation de savoir-faire spécifiques, organisation sociale de la production. Il prétend expliciter et objectiver les liens entre la typicité du produit et celle de son terroir. L'obligation de définir des règles de production précises et de délimiter strictement l'aire de l'appellation, le pilotage de la démarche puis la validation de l'appellation par le Ministère de l'Agriculture, l'engagement collectif et solidaire des producteurs à respecter un cahier des charges, font de l'AOC un modèle de référence en ce qui concerne la valorisation du terroir.
Les initiatives regroupées dans le modèle « entreprise de terroir» ont un caractère individuel. Elles émanent d'agriculteurs, d'artisans, de PME, qui commercialisent un ou plusieurs produits de la zone dans laquelle ils sont implantés. La qualité des produits, sauf quand il s'agit de produits sous signe officiel de qualité, est rarement reliée de manière explicite aux caractéristiques du territoire. L'entreprise se contente de simples allégations qui renvoient au mode de production qualifié d'« artisanal», de « traditionnel », de « fermier », ou aux grandes familles d'aménités du territoire, histoire, patrimoine bâti, nature, etc. L'ancrage de l'entreprise (ancienneté de son implantation, relations avec les producteurs) est toujours mobilisé, jouant en quelque sorte une fonction de marque. La Comtesse du Barry, par exemple, est une entreprise qui pratique la vente par correspondance de foies gras et de plats cuisinés. Son siège est situé à Gimont (Gers). La communication de l'entreprise pour les foies gras (www.comtessedubarry. com) met en avant la tradition gersoise, l'utilisation de souches authentiques, un gavage au maïs blanc. La firme crédibilise ses annonces en mentionnant l'ancienneté de son implantation en territoire de production (fondation en 1908) et en indiquant la liste nominative de ses fournisseurs de foie gras. Elle mentionne le Label Rouge et la certification «Excellence Gers » de ses produits.

Le modèle «panier de biens territorial » correspond à des démarches collectives initiées par des institutions publiques ou para publiques. Elles associent sous une même marque un ensemble de produits issus d'un territoire, qui correspond généralement à un découpage institutionnel: Pays, Département, Région. Les produits du panier doivent respecter un cahier des charges dont les clauses sont peu contraignantes. La marque territoriale fonctionne comme une marque ombrelle: elle vise à associer aux images propres à chaque produit celles, supposées positives, du territoire. Mais plus celui-ci est grand, plus les images territoriales attachées sont générales, moins ses caractères distinctifs apparaissent et plus la «valeur» qu'il apporte aux produits du panier est modeste. La marque «Pays Cathare » illustre ce modèle. Propriété du Conseil Général de l'Aude (www.cg11.fr), ses cahiers des charges ont été définis par les professionnels en relation avec les chambres consulaires, les représentants des consommateurs et les collectivités territoriales. Selon ses promoteurs, la marque est aujourd'hui utilisée par environ 500 professionnels appartenant à 20 secteurs d'activité. Ainsi sont certifiés à la fois des services (hébergement, restauration, artisanat) et des produits agroalimentaires (agneau, porc, bœuf, volailles, miel, lait, pain).

Les initiatives regroupées dans un modèle "solidaire " ont toutes un caractère collectif. Leur spécificité est qu'elles associent, sous des formes variées, des producteurs à des consommateurs. Certaines de ces initiatives (par exemple les AMAP) valorisent «la proximité » qui permettrait aux consommateurs de trouver des produits qu'ils recherchent: frais et de saison, ne supportant que des coûts de conditionnement et de transport bas, utilisant des circuits courts. La proximité favorise l'interconnaissance et 
permet aux consommateurs d'intervenir sur la construction de l'offre. Les partenaires de ces initiatives partagent souvent une même vision de l'agriculture, qu'ils souhaitent plus respectueuse de l'environnement, plutôt composée de petites exploitations et bénéficiant d'échanges plus équilibrés. La qualité se forme donc au sein d'un réseau parce que les partenaires partagent un certain nombre de valeurs mais elle n'entretient plus de liens avec le terroir. Dans d'autres initiatives, l'éloignement associé à ces mêmes valeurs, même s'il ne permet plus des relations directes, peut avoir une fonction semblable à la proximité. Le « ailleurs » devient proche, car consommer « équitable» ce serait à la fois aider les petits producteurs, consommer "généreux» et bénéficier de produits de qualité.

Différents cas peuvent illustrer ce modèle. L'AMAP des Feuillantines (www.amap.feuillantines.free.fr), créée en 2006, rassemble un groupe de consommateurs du centre de Paris et un maraîcher des alentours de Beauvais. La première distribution de légumes a lieu au mois de mai 2006 dans un magasin "artisans du monde». Le maraîcher partenaire en phase de conversion vers l'agriculture biologique, doit rassurer à ce sujet quelques adhérents sur la qualité de ses produits en disant de son exploitation « qu'il s'agissait d'une terre familiale toujours cultivée sainement ... ». Les membres de l'AMAP insistent sur la qualité des légumes qui sont «frais, de saison, diversifiés, cultivés sans produits chimiques, disponibles au fur et à mesure qu'ils mûrissent» et sur le fait que la proximité de la ferme minimise les transports et les emballages.

Le label «Genève Région» (www.terre-avenir.ch), destiné à promouvoir la consommation des produits agricoles genevois par les Genevois (www.lecourrier.ch), a été élaboré en collaboration par des agriculteurs, des distributeurs, des consommateurs et des syndicats. Il suit quatre lignes directrices: la proximité (renforcer la confiance entre producteurs et consommateurs, réduire les coûts de transports, entretenir le paysage rural, maintenir la biodiversité), la qualité (fraîcheur, diversité, respect de l'environnement), l'équité (prix négociés et rémunérateurs, bonnes conditions de travail tout au long de la filière) et la traçabilité.

Enfin, le modèle «spécialité industrielle locale " est mis en œuvre par les acteurs de l'agroalimentaire, quel que soit le degré et le stade de la transformation. La matière première agricole utilisée est homogène (homogénéisée) et standardisée. Elle s'échange sur le marché ou par le biais d'engagements (normes, contrats, intégration) liant producteur (ou groupement de producteurs) et industriel. La qualité reconnue au produit agricole passe alors par la constance de ses caractéristiques et par son adaptation aux traitements technologiques, aux modes de stockage et au conditionnement de masse, notamment. Elle est recherchée par des acteurs qui la mesurent au gré des facteurs agro-écologiques (sols,micro-climat, etc.), techniques ou identitaires qu'ils confèrent aux espaces productifs repérés. Dès lors, le lien au territoire construit par l'agriculture industrielleest ténu mais avéré: que ce soit en grandes cultures (blés, sojas, maïs, etc.) ou pour des productions de masse destinées à des marchés internationaux très normatifs (exemple des filières vins d'exportation sud-américaines), il engage les acteurs des filières dans une sélection du bien agricole selon l'origine géographique, autrement dit selon un terroir synonyme de milieu productif spécifique, et de facteurs qualitatifs intrinsèques. Les blés obtenus en climat sec et repérés comme plus aptes à la fabrication de pâtes, ou les sojas retenus en fonction d'une teneur protéinique plus importante, révèlent, par exemple, la connaissance fine qu'ont les opérateurs agro-industriels d'espaces productifs finalement moins homogènes qu'il n’y paraît (Beauce, Pampa argentine, Cerrados brésiliens, par exemple). Si les attributs qualifiant la matière agricole concernée restent difficilement identifiables et identifiés par le consommateur final, il n'en reste pas moins que le modèle industriel s'empare lui aussi du territoire, dans un lien fort et, en même temps, moins visible. Ce dernier définit une qualité technique, efficiente, rationalisée, base même de la production agroalimentaire de masse.

Les cinq modèles ainsi présentés se déclinent en un grand nombre de variantes; la figure 3 en donne quelques exemples marquants. L'IGP, les mentions « Montagne» ou «Produits-pays» peuvent ainsi se concevoir comme des déclinaisons du modèle « AOC ». Les AMAP, les labels de proximité ou ceux du commerce équitable apparaissent comme des variantes du modèle "solidaire», tandis que la mention «Fermier»s'apparente au modèle « entreprise de terroir ». Le Label rouge, les certifications de conformité du produit apparaissent pour leur part comme des variantes du modèle «spécialité industrielle locale». Quant au modèle « panier de biens », outre les marques territoriales et les marques collectives de producteurs, il trouve également une variante chez certaines marques de distributeur. Mais alors que dans les démarches précédentes il s'agissait pour des entreprises du territoire d'écouler des produits locaux, les distributeurs souhaitent, quant à eux, répondre à l'évolution de la demande en construisant des gammes de produits de terroir. Cette construction de l'offre passe alors par la sélection de produits au sein du gisement que représentent les terroirs. L'espace de la marque devient le pays entier et la qualité territorialisée ne peut être qu'évoquée au travers de références très généralesà l'histoire, au patrimoine bâti, aux savoir-faire traditionnels, à la richesse du patrimoine culinaire. L'argument traditionnel de la grande distribution, à savoir proposer des produits à un prix plus bas que les concurrents, reste utilisépour ces MDD de terroir.

$\mathrm{Au}$ fil de ces déclinaisons apparaissent des initiatives «hybrides », qui empruntent à plusieurs modèles à la fois, posant ainsi la question des convergences entre des démarches qui ont pourtant pour objectif la différenciation des produits. 


\section{LES MODĖLES DE LA QUALITÉ TERRITORIALISÉE ET LEURS DÉCLINAISONS}

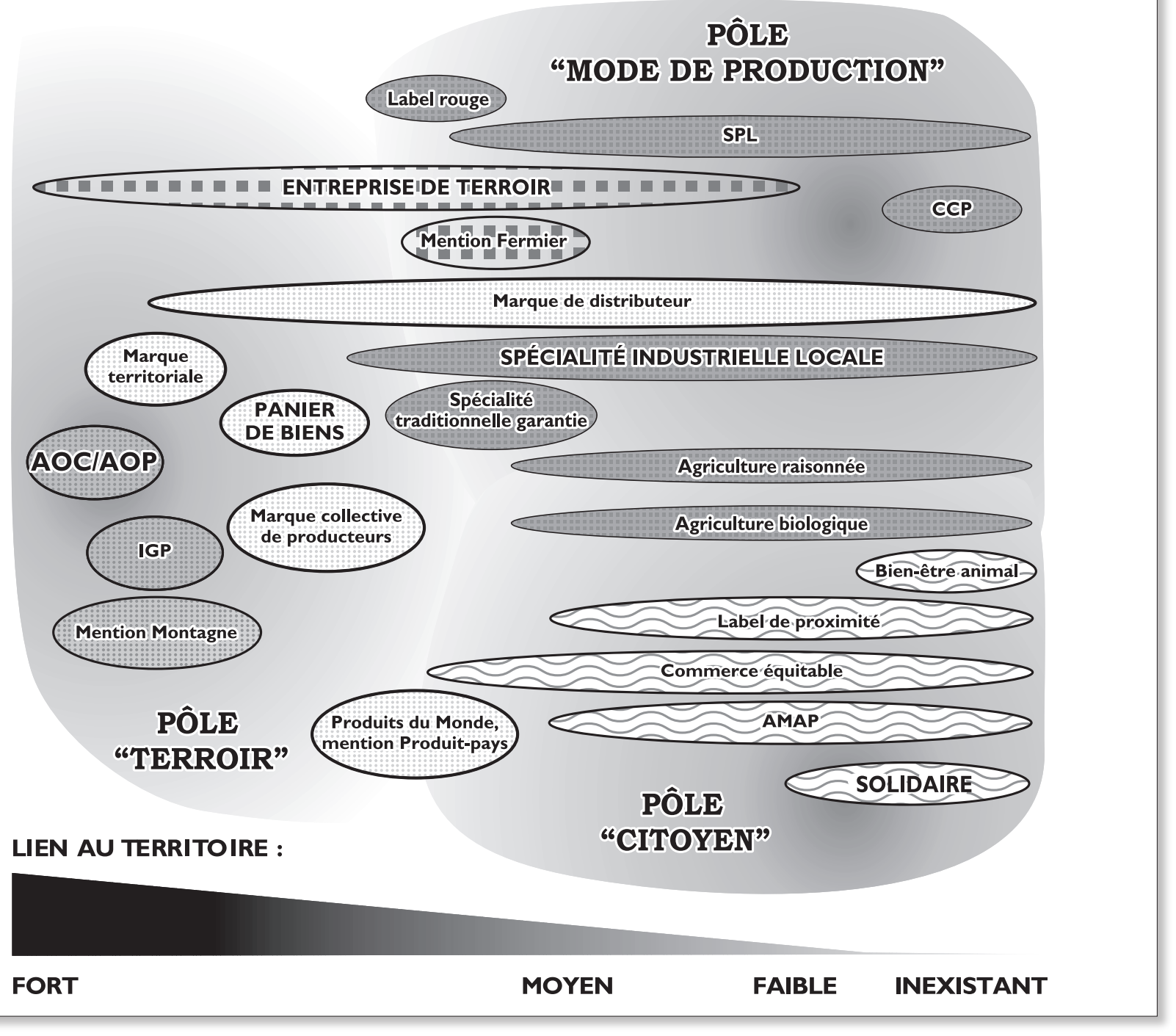

FIG. 3 - AMAP: Association pour le Maintien d'une Agriculture Paysanne, CCP: Certification de Conformité du Produit, IGP: Indication Géographique Protégée, SPL: Système Productif Localisé.

\section{4 - Convergence et divergence des démarches de qualité territorialisée}

Les stratégies qui visent à différencier un produit, en affichant ses qualités spécifiques, se doublent de stratégies d'imitation, pour «suivre» les démarches couronnées de succès. Le recours à la théorie économique apporte quelques éléments pour interpréter ce mouvement.

\section{I - Différenciation et mimétisme}

Les cas concrets que nous avons analysés s'inscrivent rarement dans un seul des trois registres de la qualité agroalimentaire (pôles) et combinent les variables (type d'initiateur, démarche collective ou non, explicitation du lien au territoire, nature de la garantie apportée au consommateur) qui nous ont permis de proposer cinq modèles d'organisation de la qualité territoriale.

Ainsi, les initiatives qui se placent dans le pôle « citoyen » mobilisent aussi les registres des pôles «mode de production » ou « terroir ». Lobodis, par exemple, grossiste qui propose une gamme de cafés certifiés équitables Max Havelaar (www.lobodis.com), met évidemment en avant ce qui inscrit le commerce équitable dans le pôle «citoyen», à savoir le «prix juste». Mais, quand la firme déclare «mettre les producteurs au cœur $\mathrm{du}$ produit en valorisant leurs traditions, leur terroir et leur savoir-faire ", ou insiste sur la notion de café de « grand cru », sa communication renvoie aux références du pôle «terroir ». Dans la logique du modèle solidaire, Lobodis établit des liens étroits avec les producteurs, par exemple au Pérou avec 1483 caféiculteurs coopérateurs péruviens de Tingo Maria, et en France avec un centre d'aide par le travail qui assure la torréfaction. La garantie donnée aux consommateurs passe aussi par ces références à des acteurs de l'économie sociale. Mais, comme dans le modèle de l'entreprise de terroir, son initiative a bien un caractère individuel et le grossiste n'hésite pas à travailler avec la grande distribution. Par ailleurs, comme le montre Eric RÉMY (2005), de nombreux producteurs de produits biologiques développent des argumentaires qui orientent leurs produits vers un «bio terroir» 
ou vers un «bio politique ». Si le consommateur attendait jusque-là d'abord des produits « sains », il est aujourd'hui soucieux, aussi, d'acheter des produits qui ne portent pas atteinte à l'environnement.

Des liens étroits peuvent de plus s'instaurer entre les démarches du pôle «mode de production» et celles du pôle « terroir ». Avigers (www.avigers.com), par exemple, commercialise des poulets sous Label Rouge et met bien entendu en avant le respect d'un certain nombre de règles de production. Mais la firme mobilise en outre des références aux aménités du département du Gers. La qualité du produit tient alors au moins autant au respect de la tradition Gasconne, à la «nature préservée gentiment balayée par le vent des Pyrénées », voire même à l' "Art de Vivre » qui fait « la richesse du Gers », qu'à la durée d'élevage, la nature de l'alimentation ou l'utilisation de parcours enherbés.

Les démarches inscrites dans le pôle «terroir» évoluent elles aussi, comme le montre la révision des cahiers des charges des produits d'AOC, qui incluent de plus en plus fréquemment des préoccupations éthiques (bien-être animal) ou renforcent leurs préoccupations sanitaires (interdiction de l'ensilage pour l'alimentation $\mathrm{du}$ bétail, dans certaines filières fromagères, ce mode d'alimentation pouvant engendrer des problèmes de qualité bactériologique du lait). Mais il manque encore à faire figurer officiellement les préoccupations de développement et de citoyenneté dans les procédures de définition des AOC. La présence de produits sous signes de qualité au sein, par exemple, des marques de distributeur de produits de terroir, témoigne également d'un métissage des modèles de la qualité territorialisée.

$\mathrm{Au}$ final, tout se passe comme si certains acteurs cherchaient à additionner les sources de rente économique attachées à chaque pôle et à chaque modèle. Les convergences se multiplient et cette évolution semble s'être accélérée depuis que les deux premiers pôles «terroir» et « mode de production », relativement anciens, semblent « bousculés » par le développement beaucoup plus récent du pôle « citoyen ».

Deux scénarios sont alors envisageables, d'une part celui de l'effondrement des démarches de qualité territorialisée, leur foisonnement-rapprochement aboutissant à un « magma » incompréhensible pour le consommateur, d'autre part, celui d'une restructuration forte, à l'issue de laquelle émergeront un nombre limité de démarches très construites et clairement lisibles. Ces éventualités renvoient toutes deux à l'instabilité des stratégies de différenciation développées par les acteurs en présence.

\section{2 - Interactions entre pôles, instabilité et convergences des modèles: essais d'interprétation}

Les pôles auxquels peuvent être apparentées les différentes formes de qualité des produits agroalimentaires ne peuvent être véritablement bornés que par des frontières floues et mouvantes, vivantes selon les jeux d'acteurs. Nous souhaitons interroger les forces d'interaction entre les pôles qui concourent à l'instabilité des modèles. Pour l'économiste, les principales forces d'interaction entre ces pôles relèvent d'une part du jeu concurrentiel entre les promoteurs-offreurs sur les marchés, d'autre part de la réaction des consommateurs en situation d'hyperchoix et, enfin, de la relation producteur-consommateur.

S'agissant du jeu stratégique des promoteurs-offreurs des modèles, la fluctuation des modèles peut s'expliquer à partir des principes de la concurrence monopolistique. La différenciation par la qualité est une stratégie de marché visant à rechercher un pouvoir de monopole (CHAMBERLIN, 1933) en se positionnant sur une « niche » de marché. Qualifier un produit à partir d'attributs originaux permet de mettre à distance les autres concurrents. Les différentes qualifications des produits conduiraient à une répartition spatiale des produits sur un marché (Hotelling, 1929). L'identification des trois pôles « terroir », « mode de production » et « citoyen » indique que l'évolution des modèles passe par une recherche d'attributs spécifiques, mais aussi positionnés de façon originale autour de ces trois pôles.

Cependant, le jeu concurrentiel qui conduit à définir des attributs supplémentaires peut aussi produire des effets inverses. En effet, l'attrait suscité pour une plus grande liberté sur les prix (la rente monopolistique) encourage l'arrivée de nouveaux concurrents sur ce même marché niche. On comprend bien ainsi pourquoi les modèles relevant du pôle «mode de production» peuvent tenter de mettre en avant des attributs relevant d'un autre pôle. C'est par exemple le cas de produits $\mathrm{AB}$ mis en scène à l'aide d'un «terroir». La convergence des modèles ne serait ainsi que le reflet d'un simple jeu concurrentiel. Mais cette convergence est-elle inéluctable?

L'entrée de nouveaux producteurs conduit à rendre excédentaires les capacités de production des offreurs en place, et à réduire leurs marges de manœuvre. Les tensions concurrentielles dépendent des degrés de voisinage et de proximité entre les attributs des produits présents sur le marché. La spécificité des produits revendiquée par les producteurs peut devenir à tout moment insuffisante pour leur permettre de dégager une réelle valeur ajoutée ou pour vendre leur production. De nouvelles spécifications doivent être sans cesse recherchées pour échapper à l'arrivée de produits substituts. Les initiatives concrètes et leur modèle évoluent en fonction d'un jeu concurrentiel dynamique et porteur d'instabilités.

Le développement des stratégies de différenciation s'inscrit par ailleurs au sein de marchés agro-alimentaires marqués par un ralentissement de la croissance de la consommation. Dans ce contexte, le comportement des consommateurs pèse davantage sur l'évolution des modèles. En effet, côté consommation, une aire de «l'hyper-choix » tendrait à s'installer (Larceneux, 2006). Comment les consommateurs peuvent-ils réagir face à la multiplication du nombre de références pour un même besoin? 
Le consommateur classe-t-il vraiment l'ensemble des produits et des attributs? -t-il une préférence pour les initiatives rattachées à l'un ou l'autre pôle ? Selon les spécialistes du marketing, les consommateurs ne compareraient pas plus de huit références dans un linéaire. De plus la reconnaissance des attributs relatifs au terroir dépendrait non seulement de la distance géographique mais aussi de la distance cognitive que les consommateurs ressentent envers les terroirs (Giraud, 2006). Il semble aussi que les consommateurs limitent le nombre des décisions quotidiennes qu'ils peuvent prendre pour leur alimentation à 14 en moyenne, alors qu'ils pourraient vraiment en prendre près de 200 (WANSINK et SoBAL, 2007). Le consommateur, en situation d'hyperchoix, s'appuie davantage sur des éléments symboliques pour effectuer ses choix. Nos trois pôles représenteraient trois symboliques fortes pour le consommateur.

La question des modes de qualification des produits reste étroitement liée aux relations producteurs- consommateurs. Economistes et sociologues ont longuement interrogé ces relations à partir du concept de conventions de qualité (Boltanski et alii., 1991). Les accords sur la qualité qui peuvent contribuer à l'émergence de modèles s'appuient sur des repères cognitifs, partagés entre producteurs et consommateurs. La justification de la qualité peut ainsi s'appuyer sur une argumentation de type industriel (savoir-faire), domestique (proximité, quotidien), marchande (prix), civique (engagement citoyen), de réseau (organisation, flexibilité), inspiré (valeurs, idéologie), de renom (réputation, ancienneté). Cet apport théorique des conventionnalistes est utile pour confirmer que notre observation des modèles renvoie généralementà des arbitrages entre plusieurs repères cognitifs. Nos pôles constituent dans ce cadre de véritables compromis: le pôle terroir relèverait ainsi d'une logique de renom, domestique, marchande. Dans cette approche, le moteur de l'évolution des modèles est la critique des modèles en place et dominants, conduisant à l'avènement de modèles alternatifs. Notre analyse des pôles et des modèles tend à dépasser l'exercice typologique, en s'appuyant sur une hypothèse relativement nouvelle dans le champ des recherches sur la qualité des produits: celle d'une interaction entre les modèles de qualification, façonnée par les attractions que peuvent exercer les pôles. L'attraction du pôle « citoyen » est-elle aujourd'hui suffisamment forte pour conduire le modèle « $\mathrm{AOC}$ » à faire valoir des attributs supplémentaires, renvoyant par exemple à des valeurs de solidarité, de citoyenneté ?

\section{5 - Conclusion}

Les évolutions en cours présentent différents risques et opportunités pour les terroirs et les démarches de qualité qui s'en réclament. Tout d'abord, les modes actuels de production et de relation entre producteurs et consommateurs reposent souvent moins sur la notion de terroir que sur celles de bassin de production, de système productif localisé, voire «d'environnement». Ils dépendent souvent moins d'une proximité géographique que d'une proximité organisationnelle et sociale.

En outre, aussi bien au niveau de la production que de la commercialisation, le jeu perpétuel des stratégies de différenciation et d'imitation implique l'invention permanente de nouvelles initiatives et impose des capacités d'adaptation rapides. Sur ces points, le modèle AOC, qui apparaissait comme le meilleur défenseur et le meilleur promoteur des terroirs, car appuyant la revendication d'une qualité spécifique du produit sur une approche large et cohérente des relations au terroir, peut paraître menacé. Les exigences réglementaires qui font la force de ce modèle sont aussi pour lui un facteur de risque. Du fait des conditions d'ancienneté et d'antériorité exigées, le gisement des AOC potentielles est limité. D'autres contraintes restreignent encore sa capacité d'adaptation, notamment face à certaines demandes des consommateurs, par exemple de disposer de produits, ou de gammes de produits, incorporant plus de services.

Le jeu des stratégies de différenciation et d'imitation a également pour conséquence un foisonnement des démarches de qualité, qui de surcroît hybrident plusieurs modèles. Sur ce point aussi, le modèle AOC peut paraître menacé. Si, jusqu'à une date récente, l'AOC était à peu près la seule alternative à la consommation de produits agroalimentaires standardisés, aujourd'hui, les alternatives sont devenues très nombreuses. La plupart mobilisent sélectivement les composantes du territoire pour promouvoir un produit, se préoccupent peu de la réalité de leurs liens avec la qualité et font référence à des échelles géographiques bien différentes de celle du terroir. Elles contribuent donc à brouiller la perception qu'ont les consommateurs de ces terroirs. Or, il a été démontré que plus les consommateurs se trouvent dans un environnement complexe, plus ils se raccrochent à des éléments symboliques, de plus en plus éloignés d'une qualité objectivable. L'attribut "origine» en paraît affaibli; le terroir semble à la fois marginalisé et «phagocyté ».

Trois enjeux se dégagent finalement, pour que les terroirs trouvent de nouvelles opportunités de développement:

- l'adaptation d'un modèle AOC relativement rigide, pris dans un environnement concurrentiel en mutation rapide; l'objectif pourrait être de développer une AOC « civique », au sein de laquelle convergeraient les attentes relatives à l'origine, aux modes de production et à la citoyenneté;

- le repositionnement d'un modèle AOC, dans ses articulations avec les autres modèles; ces derniers pourraient être pour les consommateurs des «introductions » à la qualité territoriale;

- la formation des consommateurs quant à la réalité des liens entre territoires et qualité des produits; des consommateurs disposant d'une culture plus solide en la matière aimeraient davantage l'inscrire dans leur quotidien et apprendraient à la réclamer; nul doute alors que les systèmes productifs sauraient s'adapter à une attente ainsi réaffirmée... 


\section{6 - Bibliographie}

Bérard L., Hirczak M., Marchenay Ph., Mollard A., Pecqueur B., (2005), « Le panier de Biens : une construction patrimoniale et territoriale, exemple de la Bresse », communication au symposium international INRA-PSDR « Territoire et Enjeux du développement régional », Lyon, 9-11 mars 2005.

Bérard L., Marchenay Ph., (2004), Les produits de terroir. Entre cultures et règlements, Paris, CNRS Éditions, 229 p.

Berges-Sennou F., (2002), Les marques de distributeurs ; état des lieux en France et réflexions économiques, INRA.

Boltanski L., ThÉvenot, L., (1991), De la justification: les économies de la grandeur, Paris, Gallimard.

Chamberlin E. H., (1933), The Theory of Monopolistic Competition, 6 édition, Cambridge, Masse, Harvard University Press.

Coquart D., Pouzenc M., (2007), «L'origine des produits, est-ce important pour les consommateurs ? à paraître in Pilleboue Jean (éd.), Parfums des territoires: les produits agro-alimentaires de qualité, PUM, 17 p.

Cova B., (1996) « Le lien plus que le bien. Rebondissement ou mutation du système de consommation », Futuribles, ${ }^{\circ}$ 214, novembre 1996.

Giraud G., (2006), «Les consommateurs et les terroirs », communication au Forum Planète Terroir - Aubrac 2006, 22-23 sept, Laguiole.

Goodman D., (2004), Rural Europe Redux ? Reflections on alternative agro-food networks and paradigm change, Sociologica Ruralis $\mathrm{n}^{\circ} 44$.

Guibert M., (2005), « Les systèmes agro-pastoraux des Pampas sud-américaines », in Medina L. et Hardy S., L’Amérique latine, Nantes, Éd. du Temps, Coll. Questions de géographie, p. 78-92.

Hotelling H., (1929), “Stability in competition”, Economic Journal n 39, p. 41-57.

Larceneux P., (2006), « Tests statistiques de l'hyperchoix et les stratégies des consommateurs », Credoc Cahier de recherche $\mathrm{n}^{\circ} 226,130 \mathrm{p}$.

MundLer P. (dir.), (2006), Fonctionnement et reproductibilité des AMAP en Rhône-Alpes, ISARA.

Olivier V., Wallet F., (2005), «Filières agro-alimentaires et développement territorial: une lecture des dynamiques de proximités institutionnelles », Revue Economie et Institutions, - numéro spécial « Proximités et Institutions: nouveaux éclairages », n 6-7, p. 75-100.

Pilleboue J., (2000), «Les nouveaux bassins de production de la qualité agro-alimentaire: clarification ou confusion territoriale? », Hommes et Terres du Nord, Villeneuve d'Ascq, n4, p202-210.

Pilleboue J., (2004), "Noms de produits, noms de pays »: magies des noms », in "Géographie des saveurs », Géographie et Cultures, $\mathrm{n}^{\circ}$ 50, p122-129.

Rastoin J.-L., Vissac-Charles V., (1998), « Le groupe stratégique des entreprises de terroir », RIPME.

RÉmy E., (2005), « Voyage en pays bio », Décisions marketing, nº 33.

Wansink B., Sobal J., (2007), “Mindless eating. The 200 daily food decision we overlook", Environment and Behaviour, vol. XXXIX, n 1 . 